\title{
Galaxy Group Properties in Observations and Simulations
}

\author{
Pasi Nurmi \\ University of Turku, \\ Department of Physics and Astronomy, Tuorla Observatory, Finland \\ email: pasnurmi@utu.fi
}

\begin{abstract}
In this project, we compare different properties of galaxy groups in cosmological N-body simulations and SDSS galaxy group catalogs. In the first part of the project (Nurmi et al. 2013) we compared the basic properties of the groups like the luminosity functions, group richness and velocity dispersion distributions and studied how good is the agreement between the mock group catalog and the SDSS group catalog. Here we continue the earlier study and use updated galaxy group catalog (SDSS DR10) and new simulation data (Guo et al. 2013). We reanalyse earlier group properties and include new properties in the analysis like group environment, star formation rates and group masses. Our analysis show that there are clear differences between the simulated and observed properties of galaxy groups, especially for small groups with a few members. Also, the high luminosities are clearly overestimated in the simulations compared with the SDSS group data.
\end{abstract}

Keywords. methods: numerical - methods: statistical - galaxies: clusters: general - cosmology: miscellaneous - large-scale structure of Universe

\section{Introduction}

Many numerical and analytical studies of the cluster scale dark matter halos agree well with observed cluster abundances. However, group environment influences the galaxy formation and galaxies have different properties in different large-scale environments. In our earlier study (Nurmi et al. 2013) we compared mock galaxy group catalogs against the galaxy groups obtained from the observations (SDSS, DR7). All group property distributions had similar shapes and amplitudes for richer groups, but for smaller groups and galaxy pairs there were clear differences. This indicates that we don't fully understand the galaxy formation in group environment. We continue the previous study and use updated simulation data and observational group catalogue.

\section{Data and analysis}

Our comparison data consists of two samples: galaxy groups from observations (SDSS, DR10) and galaxy groups from the simulations. The simulated galaxies are taken from the Millennium simulation database and the simulation is based on the updated version of the Millennium simulation (Springel et al. 2005). The simulation follows the evolution of $2150^{3}$ particles from the redshift $z=127$ in a box of $500 h^{-1} \mathrm{Mpc}$ on a side and the cosmological parameters of the simulation agree with WMAP7 cosmology: $\Omega_{\mathrm{m}}=\Omega_{\mathrm{dm}}+$ $\Omega_{\mathrm{b}}=0.27, \Omega_{\mathrm{b}}=0.045, h=0.70, \Omega_{\lambda}=0.73, n=0.96$, and $\sigma_{8}=0.81$. The simulated galaxy evolution is based on merger trees and galaxy properties are obtained by using semi-analytical galaxy formation models, where the star formation and its regulation by feedback processes are parameterized in terms of analytical physical models (Guo et al. 2013). From their catalogue, we use the $r$-band magnitudes that include the dust 


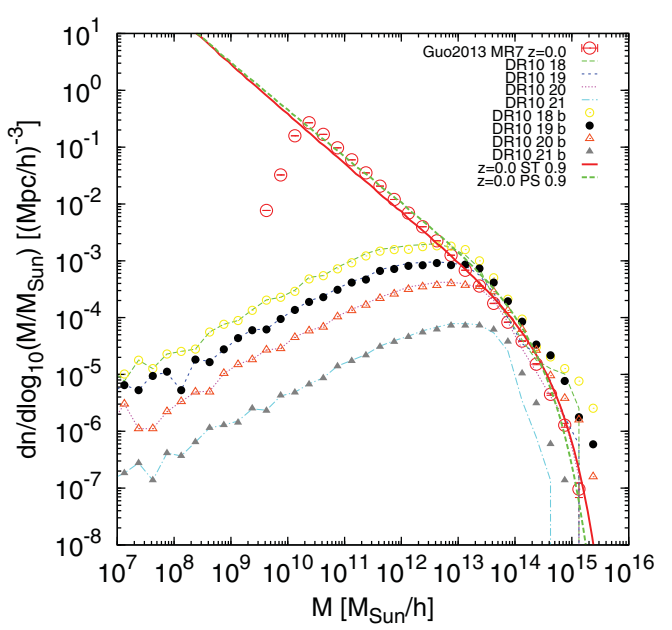

Figure 1. The mass functions of groups in different volume limited samples. Two different mass estimations of groups are shown. Guo2013 refers to mass function calculated from the virial masses of the DM halos in simulations. Also analytical mass functions by Sheth \& Tormen (1999) and Press \& Schechter (1974) are given.

extinction. The observed galaxies and galaxy groups come from the SDSS DR10 (Tempel et al. 2014).

\section{Results}

As a first test, we calculated the luminosity functions of all galaxies in the simulations and observations. The agreement is good except for large luminosities that are notably overestimated in the simulations. The agreement has been improved from one simulation to another, but clear differences are still seen for galaxies with $M<-22.5$. The Fig. 1 shows the mass functions of the galaxy groups obtained from the observations. These are shown together with the virial mass function of DM halos obtained from the Quo et al. (2013) simulation. Although the luminosity functions do not agree, the mass functions are in better agreement for cluster size objects. Group masses start to deviate from the halo mass function due to the incompleteness of the observed galaxies. This occurs for groups with $M_{t o t}<10^{13} \mathrm{~h}^{-1} M_{\text {Sun }}$ that may be the border beyond which all the groups are not virialized and the assumptions fail. We also studied the richness distribution of groups in DR10 and mock data. For galaxy pairs and small groups with $<10$ members the abundances agree well, but in the simulation there are less rich groups ( $\mathrm{n}$ of galaxies $>10$ ). This can be due to the problems in the grouping-algorithm or then the galaxies in the SAM groups are distributed in the different way.

\section{References}

Guo, Q., White, S., Angulo, R. E., et al. 2013, MNRAS, 428, 1351

Nurmi, P., Heinämäki, P., Sepp, T., et al. 2013, MNRAS, 439, 380

Press, W. H. \& Schechter, P. 1974, ApJ, 187

Sheth, R. \& Tormen, G. 1999, MNRAS, 308

Springel, V., White, S., Jenkins, A., et al. 2005, Nature, 435, 629

Tempel, E., Tamm, A., Gramann, M., et al. 2014, A\& $A$, 566, A1T 